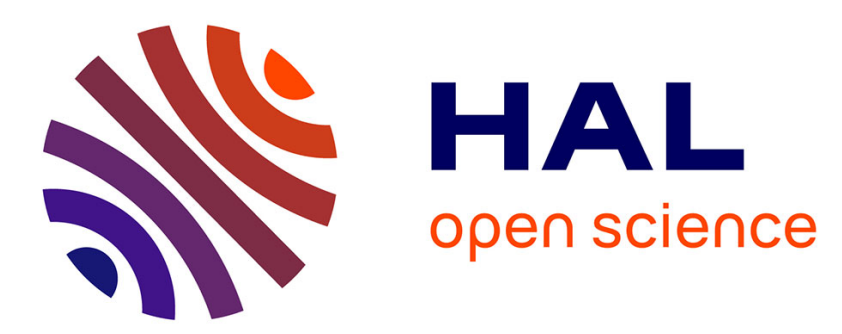

\title{
A Density Functional Theory based Thermodynamic model of Hydrogen Coverage on the W(110) Surface
}

Z A Piazza, M Ajmalghan, R D Kolasinski, Y. Ferro

\section{To cite this version:}

Z A Piazza, M Ajmalghan, R D Kolasinski, Y. Ferro. A Density Functional Theory based Thermodynamic model of Hydrogen Coverage on the W(110) Surface. Physica Scripta, 2020, T171, pp.014025. 10.1088/1402-4896/ab4474. hal-02523047

\section{HAL Id: hal-02523047 \\ https://hal.science/hal-02523047}

Submitted on 28 Mar 2020

HAL is a multi-disciplinary open access archive for the deposit and dissemination of scientific research documents, whether they are published or not. The documents may come from teaching and research institutions in France or abroad, or from public or private research centers.
L'archive ouverte pluridisciplinaire HAL, est destinée au dépôt et à la diffusion de documents scientifiques de niveau recherche, publiés ou non, émanant des établissements d'enseignement et de recherche français ou étrangers, des laboratoires publics ou privés. 


\title{
A Density Functional Theory based Thermodynamic model of Hydrogen Coverage on the W(110) Surface
}

\author{
Z. A. Piazza ${ }^{a}$, M. Ajmalghan ${ }^{a}$, R.D. Kolasinski ${ }^{b}$ and Y. Ferro ${ }^{a}$ \\ áLaboratorie PIIM, Aix-Marseille Université/CNRS, Avenue escadrille Normandie-Niemen, 13397 Marseille, France \\ b Sandia National Laboratories, Energy Innovation Department, Livermore, CA 94 551, USA
}

\begin{abstract}
Tungsten will be used as a plasma facing material in the next generation of fusion reactors. To aid in understanding atomic scale $\mathrm{H}-\mathrm{W}$ interactions, we investigated hydrogen coverage on the tungsten (110) surface via periodic density-functional theory, providing the most stable configurations that hydrogen forms on the surface at coverage ratios of interval 0.25 , step-wise, up to a full mono-layer of hydrogen. We then calculate the Gibbs free energy for the stable configurations in the presence of hydrogen gas at specified temperature and pressure. It follows that the configuration, and corresponding coverage ratio, which yields the lowest Gibbs free energy is used to estimate the macroscopic surface state. Our findings based on the model compare well to low energy electron diffraction (LEED) measurements, primarily the presence of well-ordered phases with coverage ratios $0.5,0.75$, and 1.0 , respectively, and that no ordered phases are expected as temperature increases and surface depletion occurs.
\end{abstract}




\section{Introduction}

In our previous work, we showed via density functional theory (DFT) and comparison with low energy ion scattering (LIES) direct recoil spectroscopy (DRS) experiments that saturation of hydrogen on the W(110) surface is expected at a coverage of 1.0 [1]. Low energy electron diffraction (LEED) and Thermal Desorption Spectroscopy (TDS) experiments on single crystalline $\mathrm{W}(110)$ also find a saturation ratio of 1.0 under vacuum pressure and room temperature conditions[2-4]. Of course, with increasing temperature, due to entropic effects which favor the gas phase, the surface coverage will lower and eventually deplete. We herein estimate the expected coverage with specific temperature and pressure conditions by comparing the Gibbs free energy of a set of experimentally observed adsorption patterns corresponding to coverage ratios from the bare surface up to 1.0 .

Due to the high melting point, high thermal conductivity, high sputtering threshold, and low tritium solubility of Tungsten (W), it has been chosen as the divertor material for the JET, WEST, and ITER tokamak fusions reactors[5-7]. Thus it is of high important to understand hydrogen-tungsten surface interactions at a fundamental level. Several experimental studies have been conducted on single crystalline samples of tungsten including TDS [8-10], LIES, DRS [11], LEED [3,4,12,13], and surface reflectance spectroscopy[14] measurements. Of these techniques, LEED and LIES/DRS are best able to probe atomic-scale phenomena on the surface.

Of note is the presence of well-ordered adsorption patterns seen in LEED measurements on the W(110) surface; more specifically, Gonchar et. al.[4], Estrup[3] and Altman et. al.[13] all observed diffraction patterns of $p(2 \times 1), p(2 \times 2)$, which correspond precisely to coverages of $0.5,0.75$, respectively and vary in intensity depending upon the overall coverage. Upon further adsorption 
of hydrogen up to the experimental saturation limit, the characteristic diffraction patterns disappear, suggesting a $p(1 \mathrm{x} 1)$ adsorption pattern at a mono-layer of coverage (with coverage ratio 1.0) that matches the symmetric pattern of the underlying surface. If the surface is prepared first at the saturated mono-layer coverage, and the temperature of the system is increased, intensity associated with the lower coverage symmetry patterns increases as a result of the desorption[4]. There is no sign of a unique ordered pattern corresponding to a coverage below 0.5 where the basic unit has a $p(2 \times 1)$ pattern[4].

While pure DFT models can provide an atomic-scale description on ideal single crystalline surfaces, they do not include temperature dependent effects. To better understand the changing state of the surface with ambient conditions, we constructed a free energy model that reflects the relative stability between the known symmetric patterns probed by LEED experiments in equilibrium with hydrogen gas at fixed temperature and pressure. The model provides a simple first estimate of the macroscopic surface coverage as a function of temperature and pressure based on a minimal atomic-scale picture using discrete coverages with fixed adsorption patterns.

\section{Methodology}

\section{1 - Density functional theory calculations}

To model hydrogen adsorbed to the W(110) surface we built a periodic DFT slab model consisting of 6 layers of tungsten and a $20 \AA$ vacuum. A top-view schematic representation is provided in figure 1. The slab allows us to investigate surface coverage ratios of interval $0.25\left(\Theta_{W_{110}}=\right.$ $\left.\frac{\# \text { of adsorbed } H}{4}\right)$. The surface model is able to represent $p(2 \times 1) \Theta=0.5, p(2 \times 2) \Theta=0.75$, and $p(1 \times 1) \Theta=1.0$ patterns predicted by LEED measurements[4,14].

We use the PBE functional[15] and Vanderbilt ultra-soft scalar-relativistic pseudo-potentials[16] for both tungsten and hydrogen atoms. The unit-cell is sampled with a grid of 11x11x1 k-points. 
More details on the DFT calculations can be found in our previous works[1,17,18]. In ref. 17 it is shown that with 6 layers of tungsten and a 20 Å vacuum the surface energy of W(110) is converged to $10 \mathrm{meV} / \AA^{2}$. Vibrational properties of the adsorbed hydrogen atoms were calculated via Density Functional Perturbation Theory (DFT-PT)[19]. All DFT and DFT-PT calculations were carried out using Quantum Espresso[20].

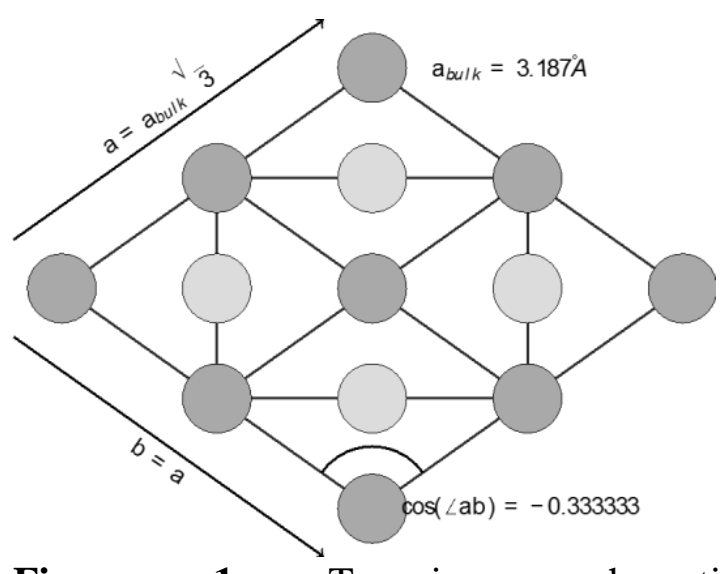

Figure 1. Top-view schematic representations of the $\mathrm{W}(110)$ surface-slab used in this work. Light grey circles represent tungsten surface atoms, white circles represent the layer beneath.

\section{2 - Gibbs energy calculations}

We consider a system of $\frac{n_{\text {tot }}}{2}$ hydrogen molecules in the gas phase in contact with a W(110) surface, where $n_{\text {tot }}$ can be an arbitrarily number, subject to the constraint that it must be greater than the saturation limit of adsorbed hydrogen atoms in the DFT working cell (taken as $n_{\text {tot }}=6$ in the present work). For a given adsorption state there are $\frac{n_{\text {tot }}-n_{\text {ads }}}{2}$ hydrogen molecules remaining in the gas phase and $n_{a d s}$ atomic hydrogen particles adsorbed to the surface. The Gibbs free energy $G(T, p)$ of the system i.e. $\frac{\left(n_{t o t}-n_{\text {ads. }}\right)}{2} \boldsymbol{H}_{\mathbf{2}}+\boldsymbol{W}_{\mathbf{1 1 0}} \boldsymbol{H}_{\boldsymbol{n}_{\text {ads }}}$ is given in equation 1: 


$$
G(T, p)=g_{W_{110} H_{n_{a d s .}}}(T)+\frac{\left(n_{t o t}-n_{a d s .}\right)}{2} g_{H_{2}}(T, p)
$$

where $g_{W_{110} H_{n}}$ is is the contribution to the Gibbs energy of the adsorbed hydrogen and the surface, $g_{\mathrm{H}_{2}}$ is the per-particle Gibbs energy of a hydrogen molecule (i.e. the chemical potential of an $\mathrm{H}_{2}$ as a function of $\mathrm{P}$ and T). $g_{H_{2}}$ is calculated with equation 2 via substitution of equations 3 and 4 :

$$
\begin{aligned}
& g_{H_{2}}(T, P)=h_{H_{2}}(T)-T \cdot s_{H_{2}}(T, P) \\
& h_{H_{2}}(T)=e_{H_{2}}^{D F T}+e_{H_{2}}^{v i b}(T)+e_{H_{2}}^{r o t}(T)+e_{H_{2}}^{\text {trans }}(T)+k_{B} T \\
& s_{H_{2}}(T, P)=s_{H_{2}}^{v i b}(T)+s_{H_{2}}^{r o t}(T)+s_{H_{2}}^{\text {trans }}(T, p)
\end{aligned}
$$

The per unit-cell Gibbs energy of an adsorbed state, $g_{W_{110} H_{n_{a d s}}}$, is calculates as:

$$
g_{W_{110 .} H_{n_{a d s}}}(T)=\left(e_{W_{110} H_{n_{a d s}}^{D F T}}^{D}+e_{W_{110}^{v i b} H_{n_{a d s}}}^{v i b}(T)\right)-T . s_{W_{110}^{v i b} H_{n_{a d s}}^{v i b}}(T)
$$

Mathematical details for the individual vibrational, rotational, and translational terms in the above expressions can be found in elsewhere [21].

The reference in energy $E_{r e f}$ was taken as the sum of the electronic energy of the bare W(110) surface $\boldsymbol{e}_{W_{110}}^{D F T}$ plus the zero-point energy corrected value $\boldsymbol{e}_{\boldsymbol{H}_{2}}^{\boldsymbol{D F T}}$ of the $\frac{\boldsymbol{n}_{\text {tot. }}}{2}$ molecules of hydrogen. By subtracting $E_{r e f}$ from $G(T, p)$, we obtain a Gibbs free energy of reaction $\Delta G(T, p)$ according to:

$$
\Delta G(T, p)=G(T, p)-\left(e_{W_{110}}^{D F T}+\frac{n_{t o t .}}{2} e_{H_{2}}^{D F T}\right)
$$

The state yielding the minimal Gibbs free energy of reaction $\Delta \mathrm{G}(\mathrm{T}, \mathrm{p})$ for a system in equilibrium under constant temperature and pressure with a fixed total number of particles corresponds to the most stable state, thus comparing $\Delta \mathrm{G}(\mathrm{T}, \mathrm{p})$ for each configuration in a set of well-chosen states provides a means to estimate the macroscopic coverage for the system.

\section{Results}




\section{$3.1-$ DFT results}

\section{1a - stable adsorption patterns}

Experimental $[3,4,13,14]$ and theoretical evidence[1] suggests that at room temperature, and low pressure conditions, the $\mathrm{W}(110)$ surface saturates at a mono-layer of hydrogen, corresponding to a coverage ratio of $\Theta=1.0$. In all published DFT works, the so-called "three-fold" (TF) site is found to be the most stable[1,22] for a single hydrogen adsorbing on the surface. LEED studies suggest that from below $30 \mathrm{~K}$ to beyond $400 \mathrm{~K}$ various well-ordered patterns emerge[3,4,13,14]. Accordingly, we found the most-stable TF adsorption patterns accessible in our unit-cell for each coverage ratio up to $\Theta=1.0$, shown schematically in Table $\mathbf{1}$ along with the corresponding perhydrogen adsorption energy calculated according to:

$$
E_{a d s, \Theta}=\left(e_{W_{W_{110}}^{D F T} H_{n_{a d s}}}^{D}-e_{W_{W_{110}}^{D F T}}^{D T}-\frac{n_{a d s}}{2} e_{H_{2}}^{D F T}\right) / n_{a d s}
$$

For a more detailed analysis of the stationary configurations that hydrogen can form up to and beyond $\Theta=1.0$, the interested reader is referred to our previous work[1].

Table 1 Top-view schematics representations of the most-stable TF configurations at intervals of 0.25 up to one mono-layer. Light grey circles represent tungsten surface atoms, white circles represent the layer beneath. Adsorbed hydrogens are represented in dark grey. Each configuration is a unit repeated periodically in space. Per-particle hydrogen adsorption energies are provided $\left(E_{a d s}\right)$ in units of eV.

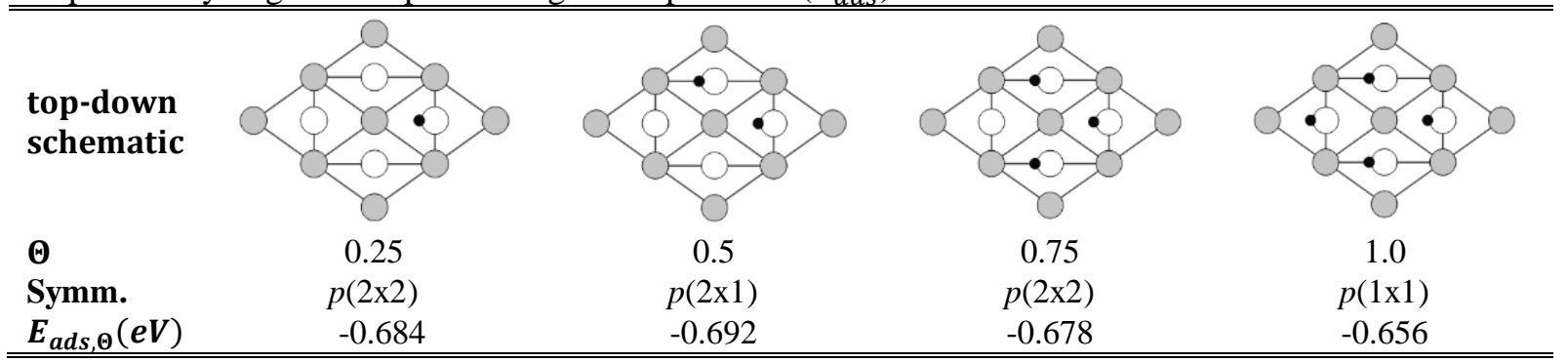

\section{$3.1 b$-energy trends}

Figures 2a and $\mathbf{2 b}$ present the trend in surface energy and adsorption energy (per adsorbed hydrogen atom) vs. increasing coverage. The surface energy is calculated as: 


$$
S E_{\Theta}=\frac{1}{2 A}\left(e_{W_{110} H_{n_{a d s}} F T}-n_{W} e_{W_{b u l k}}-\frac{n_{a d s}}{2} e_{H_{2}}^{D F T}\right)
$$

Where $A$ is the surface area of our slab model $\left(30.47 \AA^{2}\right), n_{W}$ is the total number of tungsten in the slab (in our case $n_{W}=24$ ), and $e_{W_{b u l k}}$ is the energy per tungsten atom in the bulk as calculated by DFT. All other symbols were previously defined.

The steady decrease of surface energy with increasing coverage means that the overall system becomes more and more stable upon continued adsorption of hydrogen up to $\Theta=1.0$ at $0 \mathrm{~K}$. The adsorption energy per hydrogen drops from $\Theta=0.25$ to $\Theta=0.5$, where the minimum is found, then increases up to $\Theta=1.0$.

In the LEED measurements of Gonchar et al., after annealing at $\mathrm{T}=120-200 \mathrm{~K}$ and cooling to $T=$ $5 K$ locally ordered patterns consistent with the $p(2 \times 1)$ structure are seen on $\mathrm{W}(110)$ at coverages as low as $\Theta \approx 0.3$ [4]. This is consistent with the $E_{\text {ads., } \Theta}$ minimum when $\Theta=0.5$. Since the $\Theta=$ $0.5 p(2 \times 1)$ pattern is the most stable-configuration on a per-hydrogen basis, when molecular adsorption takes place on a bare surface, an adsorbed $\mathrm{H}_{2}$ molecule would most favorably form the $p(2 \times 1)$ pattern locally as opposed to a more dispersed pattern such as the $\Theta=0.25 p(2 \times 2)$ pattern, even when $\Theta<0.5$.

Considering coverage $\Theta \geq 0.75$, the local electronic potential near a TF site is effectively different than that of a less-saturated surface as is seen by the increase in $E_{a d s ., \Theta}$ corresponding to a loss in stability per hydrogen atom at $0 \mathrm{~K}$. The same trend continues at $\Theta=1.0$ where there is a full mono-layer of hydrogen and the saturation coverage has been reached according to single crystalline LEED[3,4], LIES [1], and TDS experiments[2]. 
(a)

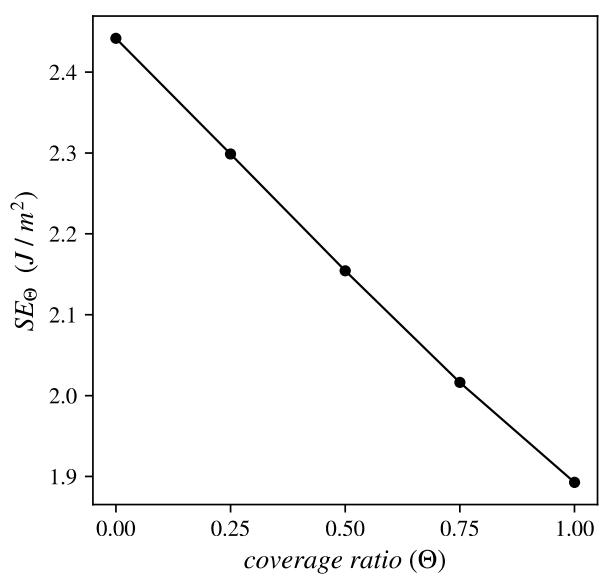

(b)

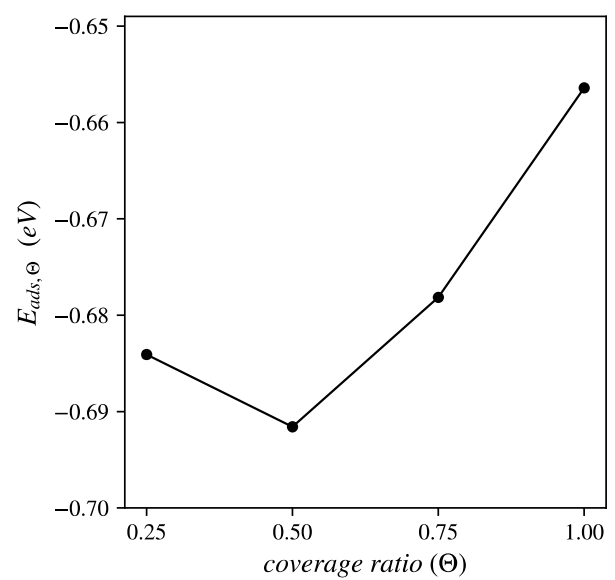

Figure 2. Energy trends of hydrogen adsorbed on the W(110) surface. (a) the surface energy $\left(S E_{\Theta}\right)$ for the most-stable adsorption patters by coverage. (b) The adsorption energy per hydrogen atom $\left(E_{a d s, \Theta}\right)$ for the W(110) surface by coverage given in eV per adsorbed hydrogen.

\section{2- Thermodynamic results}

By fixing the total number of hydrogen atoms in the system $n_{\text {tot }}$ and calculating $\Delta G(T, p)$ for each adsorption configuration according to equation 6, we can arrive at an estimate of the experimental surface state. Since the Gibbs free energy of the system is minimal at equilibrium under constant temperature and pressure with a fixed total number of particles, the corresponding adsorption configuration $(\Theta=0.0, \Theta=0.25 p(2 \times 2), \Theta=0.5 p(2 \times 1), \Theta=0.75 p(2 \times 2)$, or $\Theta=1.0 p(1 \times 1))$ with the lowest $\Delta G(T, p)$ serves as an estimate of the macroscopic adsorption state.

Figure 3a provides a set of plots of $\Delta G(T, p)$ vs. $\Theta$ at different temperatures, all with $P=$ $5.5 \times 10^{-5} \mathrm{~Pa}$. This pressure was chosen since it is within the range of surface analysis experiments[3,11]. Focusing on the minimum value of $\Delta G(T, p)$ with respect to the various isotherms shown in Figure 3a, we can see how the model yields the expected coverages plotted in Figure 3b. Mono-layer coverage $\Theta=1.0 p(1 \times 1)$ is predicted as more stable than the $\Theta=0.75$ $p(2 \times 2)$ configuration up to around $400 \mathrm{~K}$ (light grey with triangle markers). The surface coverage then drops somewhat quickly with respect to temperature; at $450 \mathrm{~K}$ (black with diamond markers) 
$\Theta=0.5 p(2 \mathrm{x} 1)$ is most stable relative to the other adsorption patterns. By $500 \mathrm{~K}$ the bare surface $\Theta=0.0$ is expected.

\section{2a-Comparison with LEED measurements}

In the LEED measurements of Gonchar et. al. [4], the relative intensity of two electron diffraction peaks, corresponding to the $\Theta=0.75 p(2 \times 2)$ and $\Theta=0.5 p(2 \times 1)$ patterns, were measured with increasing temperature after a complete mono-layer $(\Theta=1.0 p(1 \times 1))$ was first adsorbed to the surface at $77 \mathrm{~K}$. The present model predicts the $\Theta=0.75 p(2 \times 2)$ as the most stable adsorption pattern near $400 \mathrm{~K}$ and $P=5.5 \times 10^{-5} \mathrm{~Pa}$ (Figure 3b); this agrees well with Gonchar et. al.'s measurements where the LEED peak corresponding to the $p(2 \times 2)$ pattern has its maximum intensity near $400 \mathrm{~K}$. The LEED peak in Gonchar et. al. corresponding to the $p(2 \mathrm{x} 1) \Theta=0.5$ pattern has maximum intensity around $450 \mathrm{~K}$, again in close agreement with the prediction of our model where near $450 \mathrm{~K}$ the $p(2 \times 1) \Theta=0.5$ pattern is most stable.

Interestingly, $\Theta=0.25 p(2 \times 2)$ is never predicted as a stable phase in the Gibbs model, nor does a LEED pattern consistent with $\Theta=0.25 p(2 \times 2)$ show up in Gonchar's measurements. Since there is no temperature having a $p(2 \times 2) \Theta=0.25$ phase with minimum $\Delta G(T, p)$ (Figure $3 \mathbf{b})$; we can hypothesize that the system should deplete to bare coverage primarily via $\mathrm{H}_{2}$ recombination of nearest neighboring adsorbed hydrogen atoms in the $p(2 \times 1) \Theta=0.5$, leaving behind localized patches of $p(2 \times 1)$ pattern at $\Theta<0.5$. Again, this is consistent with the finding of local $p(2 \times 1)$ order in LEED measurements even below coverage 0.5 after annealing [4], however the present method is not directly capable to model such adsorption patterns.

In Gonchar's measurements, by $500 \mathrm{~K}$, the intensity of the diffraction peaks corresponding to both the $\Theta=0.75 p(2 \times 2)$ and $\Theta=0.5 p(2 \times 1)$ patterns are reduced to zero, suggesting that the surface is either fully or nearly depleted of hydrogen. This is in strong agreements with our model, where 
the surface is predicted to be bare at $500 \mathrm{~K}$ unless the background pressure of molecular hydrogen is on the order of $\sim 10^{-3} \mathrm{~Pa}$ or greater.

(a)

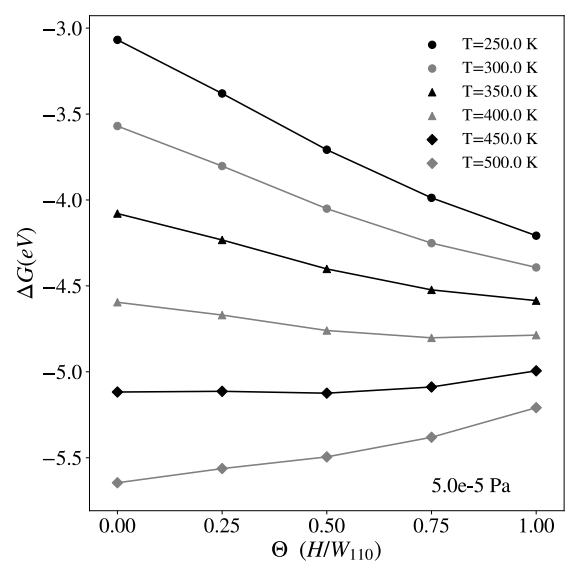

(b)

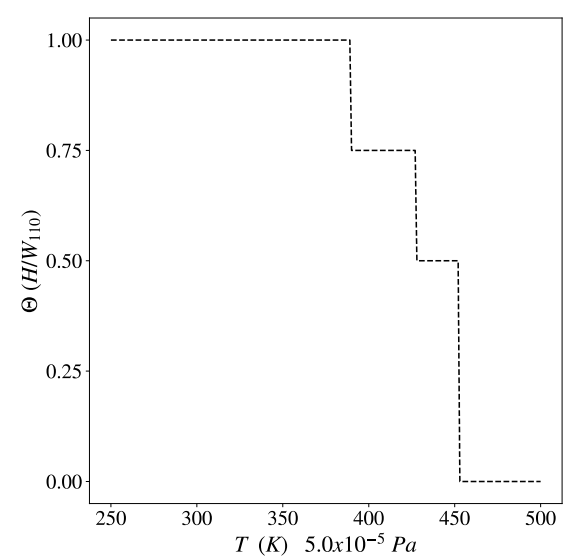

Figure 3. Temperature dependent coverage on the $\mathrm{W}(110)$ surface at $P=5.5 \times 10^{-5} \mathrm{~Pa}$. (a) Gibbs free energy of reaction (eq. 6) for each $\Theta$ at various temperatures. Connecting lines between different values of $\Theta$ at the same temperature are to aid the eye and do not represent calculated values. (b) Predicted coverage $(\Theta)$ vs. temperature.

\section{Conclusions and Perspectives}

Herein, we calculated the most stable three-fold (TF) adsorption patterns of hydrogen on the $\mathrm{W}(110)$ via DFT at coverages $\Theta=0.25,0.5,0.75, \& 1.0$. The ordered TF patterns at $\Theta=$ $0.5,0.75, \& 1.0$ correspond directly to the $p(2 \times 1), p(2 \times 2)$, and $p(1 \times 1)$ patterns measured in lowenergy electron diffraction (LEED) experiments from the literature; at $\Theta=0.25$, ordered TF adsorption corresponds to a $p(2 \times 2)$ pattern not seen directly in LEED measurements. A temperature and pressure dependent free energy model was built to investigate the relative stability of the ordered patterns under experimental conditions. Our model successfully estimates characteristics of the coverage state seen in LEED experiments as a function of the temperature under vacuum pressure, particularly the onset of hydrogen depletion around $375 \mathrm{~K}$, complete depletion (a bare surface) near $500 \mathrm{~K}$, and that no ordered $\Theta=0.25 p(2 \times 2)$ pattern is predicted at any temperature. 
While the current model captures the aforementioned experimental features, it estimates the coverage at discrete intervals associated with the ideal adsorption patterns experimentally measured on clean W(110) surfaces; however, intermediate coverage ratios are indeed seen experimentally rather than discrete phase changes. The intermediate coverage patterns are expected to be composed primarily of patch-like combinations of the $p(2 \times 2) \Theta=0.75$ and $p(2 \times 1)$ $\Theta=0.5$ patterns. In an upcoming study we construct and analyze a model that describes the surface state via a full partition function which includes the effect of higher energy adsorption configurations and allows for intermediate coverage ratios.

\section{Acknowledgements}

This work has been carried out within the framework of the French Federation for Magnetic Fusion Studies (FR-FCM) and of the Eurofusion consortium, and has received funding from the Euratom research and training program 2014-2018 and 2019-2020 under grant agreement No 633053. This work was performed under EUROfusion WP PFC. This work also received fund within the framework of the A*MIDEX project (Grant No. ANR- 11-IDEX-0001-02) funded by the Investissements d'Avenir French Government program, managed by the French National Research agency. The views and opinions expressed herein do not necessarily reflect those of the European Commission. The authors of this paper were granted access to the high-performance computing resources of IDRIS and CINES under Allocation No. A0040806612 made by Grand Equipement National de Calcul Intensif and to the Marconi Supercomputer at CINECA Super Computing Application and Innovation Department, Bologna, Italy. 


\section{References}

[1] Z.A. Piazza, M. Ajmalghan, Y. Ferro, R.D. Kolasinski, Saturation of tungsten surfaces with hydrogen: A density functional theory study complemented by low energy ion scattering and direct recoil spectroscopy data, Acta Materialia. 145 (2018) 388-398. doi:10.1016/j.actamat.2017.12.029.

[2] P.W. Tamm, L.D. Schmidt, Binding States of Hydrogen on Tungsten, The Journal of Chemical Physics. 54 (1971) 4775-4787. doi:10.1063/1.1674753.

[3] J.W. Chung, S.C. Ying, P.J. Estrup, Reconstruction of the W(110) surface induced by hydrogen adsorption, Phys. Rev. Lett. 56 (1986) 749-752. doi:10.1103/PhysRevLett.56.749.

[4] V.V. Gonchar, O.V. Kanash, A.G. Naumovets, A.G. Fedorus, Two-dimensional lattices of adsorbed hydrogen on the (011) face of tungsten and their disordering, JETP Lett. 28 (1979) 330-333.

[5] G.F. Matthews, M. Beurskens, S. Brezinsek, M. Groth, E. Joffrin, A. Loving, M. Kear, M.-L. Mayoral, R. Neu, P. Prior, V Riccardo, F. Rimini, M. Rubel, G. Sips, E. Villedieu, P. de Vries, M.L. Watkins, E.-J. contributors, JET ITER-like wall-overview and experimental programme, Phys. Scr. 2011 (2011) 014001. doi:10.1088/0031-8949/2011/T145/014001.

[6] V. Philipps, Ph. Mertens, G.F. Matthews, H. Maier, Overview of the JET ITER-like Wall Project, Fusion Engineering and Design. 85 (2010) 1581-1586.

doi:10.1016/j.fusengdes.2010.04.048.

[7] J. Bucalossi, M. Missirlian, P. Moreau, F. Samaille, E. Tsitrone, D. van Houtte, T. Batal, C. Bourdelle, M. Chantant, Y. Corre, X. Courtois, L. Delpech, L. Doceul, D. Douai, H. Dougnac, F. Faïsse, C. Fenzi, F. Ferlay, M. Firdaouss, L. Gargiulo, P. Garin, C. Gil, A. Grosman, D. Guilhem, J. Gunn, C. Hernandez, D. Keller, S. Larroque, F. Leroux, M. Lipa, P. Lotte, A. Martinez, O. Meyer, F. Micolon, P. Mollard, E. Nardon, R. Nouailletas, A. Pilia, M. Richou, S. Salasca, J.-M. Travère, The WEST project: Testing ITER divertor high heat flux component technology in a steady state tokamak environment, Fusion Engineering and Design. 89 (2014) 907-912. doi:10.1016/j.fusengdes.2014.01.062.

[8] J.P. Roszell, J.W. Davis, A.A. Haasz, Temperature dependence of deuterium retention mechanisms in tungsten, Journal of Nuclear Materials. 429 (2012) 48-54.

doi:10.1016/j.jnucmat.2012.05.018.

[9] M. Poon, A.A. Haasz, J.W. Davis, R.G. Macaulay-Newcombe, Impurity effects and temperature dependence of D retention in single crystal tungsten, Journal of Nuclear Materials. 313-316 (2003) 199-203. doi:10.1016/S0022-3115(02)01445-9.

[10] R. Bisson, S. Markelj, O. Mourey, F. Ghiorghiu, K. Achkasov, J.-M. Layet, P. Roubin, G. Cartry, C. Grisolia, T. Angot, Dynamic fuel retention in tokamak wall materials: An in situ laboratory study of deuterium release from polycrystalline tungsten at room temperature, Journal of Nuclear Materials. 467 (2015) 432-438. doi:10.1016/j.jnucmat.2015.07.028.

[11] R.D. Kolasinski, Analysis of hydrogen adsorption and surface binding configuration on tungsten using direct recoil spectrometry, Journal of Nuclear Materials. 463 (2015) 1053-1056. doi:10.1016/j.jnucmat.2014.11.115.

[12] P.J. Estrup, J. Anderson, Chemisorption of Hydrogen on Tungsten (100), The Journal of Chemical Physics. 45 (1966) 2254-2260. doi:10.1063/1.1727919. 
[13] M. Altman, J.W. Chung, P.J. Estrup, J.M. Kosterlitz, J. Prybyla, D. Sahu, S.C. Ying, Phase transformations of the H/W(110) and H/Mo(110) surfaces, Journal of Vacuum Science \& Technology A. 5 (1987) 1045-1048. doi:10.1116/1.574182.

[14] G.B. Blanchet, P.J. Estrup, P.J. Stiles, A study of the H/W(110) adsorption system by surface reflectance spectroscopy, Phys. Rev. B. 23 (1981) 3655-3668.

doi:10.1103/PhysRevB.23.3655.

[15] J.P. Perdew, K. Burke, M. Ernzerhof, Generalized Gradient Approximation Made Simple, Phys. Rev. Lett. 77 (1996) 3865-3868. doi:10.1103/PhysRevLett.77.3865.

[16] D. Vanderbilt, Soft self-consistent pseudopotentials in a generalized eigenvalue formalism, Phys. Rev. B. 41 (1990) 7892-7895. doi:10.1103/PhysRevB.41.7892.

[17] N. Fernandez, Y. Ferro, D. Kato, Hydrogen diffusion and vacancies formation in tungsten: Density Functional Theory calculations and statistical models, Acta Materialia. 94 (2015) 307-318. doi:10.1016/j.actamat.2015.04.052.

[18] M. Ajmalghan, Z. Piazza, E. Hodille, Y. Ferro, Surface coverage dependent mechanisms for the absorption and desorption of hydrogen from the W(110) and W(100) surfaces: a DFT investigation., Nucl. Fusion. (2019). doi:10.1088/1741-4326/ab33e7.

[19] S. Baroni, S. de Gironcoli, A. Dal Corso, P. Giannozzi, Phonons and related crystal properties from density-functional perturbation theory, Rev. Mod. Phys. 73 (2001) 515-562. doi:10.1103/RevModPhys.73.515.

[20] P. Giannozzi, S. Baroni, N. Bonini, M. Calandra, R. Car, C. Cavazzoni, Davide Ceresoli, G.L. Chiarotti, M. Cococcioni, I. Dabo, A.D. Corso, S. de Gironcoli, S. Fabris, G. Fratesi, R. Gebauer, U. Gerstmann, C. Gougoussis, Anton Kokalj, M. Lazzeri, L. Martin-Samos, N.

Marzari, F. Mauri, R. Mazzarello, Stefano Paolini, A. Pasquarello, L. Paulatto, C. Sbraccia, S. Scandolo, G. Sclauzero, A.P. Seitsonen, A. Smogunov, P. Umari, R.M. Wentzcovitch, QUANTUM ESPRESSO: a modular and open-source software project for quantum simulations of materials, J. Phys.: Condens. Matter. 21 (2009) 395502. doi:10.1088/0953-

8984/21/39/395502.

[21] E.A. Hodille, N. Fernandez, Z.A. Piazza, M. Ajmalghan, Y. Ferro, Hydrogen supersaturated layers in H/D plasma-loaded tungsten: A global model based on thermodynamics, kinetics and density functional theory data, Physical Review Materials. 2 (2018). doi:10.1103/PhysRevMaterials.2.093802.

[22] A. Nojima, K. Yamashita, A theoretical study of hydrogen adsorption and diffusion on a W(1 1 0) surface, Surface Science. 601 (2007) 3003-3011. doi:10.1016/j.susc.2007.05.019. 